\title{
Use of endocytoscopy in the diagnosis of a rare, depressed-type ileal adenoma
}

An 80-year-old man was admitted to hospital with adhesive ileus, which responded to conservative management. Total colonoscopy was subsequently undertaken to investigate his symptoms. There was an incidental reddish, depressed lesion with marginal elevation, $9 \mathrm{~mm}$ in diameter, in the terminal ileum. Chromoendoscopic view with indigo carmine dye showed a star-shaped demarcation line in the depressed lesion ( $\bullet$ Fig. 1 ). The morphological appearance mimicked a depressed-type colorectal cancer as demonstrated by Kudo [1]. Narrow band imaging (NBI) showed a well-demarcated lesion and NBI with magnification demonstrated regularly arranged network vessels [2] $(\bullet$ Fig. 2). A magnifying endoscopic view with crystal violet staining revealed small round and tubular pit patterns resembling colon cancer within the depressed lesion, instead of villi [3] ( Fig. 3). As the patient was anticoagulated, endocytoscopy was performed for further evaluation of the lesion in order to make a decision about further management. Endocytoscopic images showed that the nuclei were fusiform and regularly arranged along the basement membrane, and the lumen was smooth and slit-like, remarkably similar to our endocytoscopic images of colonic adenoma and intramucosal neoplasia ( $\bullet$ Fig. 4). Furthermore, these findings were not seen in the villous mucosa of the small intestine. Therefore, the lesion was diagnosed as being either adenoma or intramucosal neoplasm of the ileum and endoscopic mucosal resection was carried out. Histopathological analysis confirmed that the lesion was a tubular adenoma with low grade atypia similar to our endocytoscopic prediction ( $\bullet$ Fig. 5 ). Endocytoscopy provides real time microscopic images in vivo during routine endoscopy and has been shown to be useful in the evaluation of lesions within the gastrointestinal tract $[4,5]$. It may be particularly useful in patients who are on anticoagulant therapy where routine biopsy for histological analysis may be hazardous and also make further endoscopic treatment more difficult due to scarring. In addition, endocytoscopy may also avoid sampling error in the biopsy.

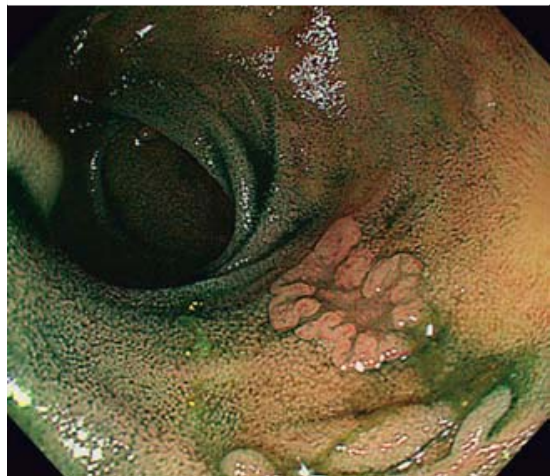

Fig. 1 Chromoendoscopic view with indigo carmine dye showed the star-shaped demarcation line of the depressed lesion.

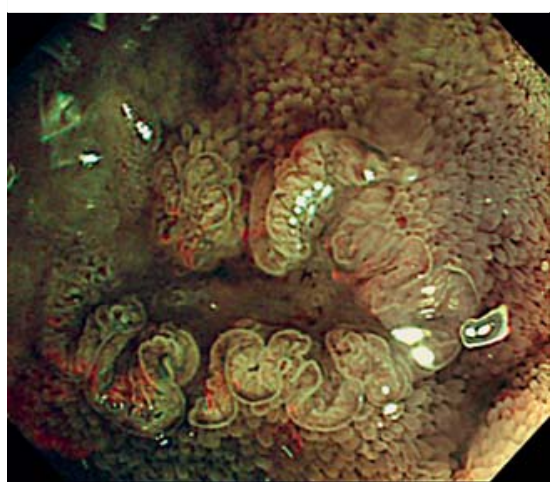

Fig. 2 Narrow band imaging (NBI) showing a well-demarcated lesion and NBI with magnification demonstrating regularly arranged network vessels.

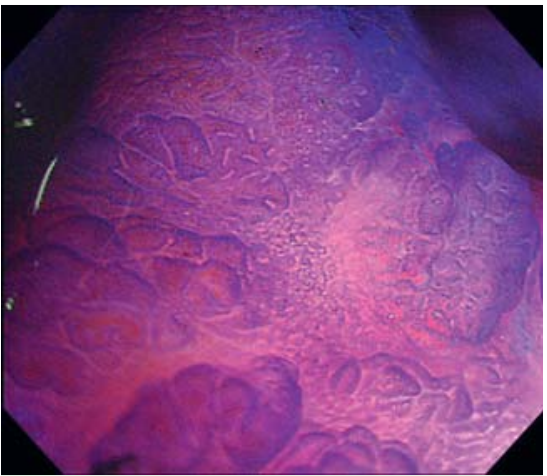

Fig. 3 A magnifying endoscopic view with crystal violet staining revealed small round and tubular pit patterns resembling colon cancer within the depressed lesion instead of villi.

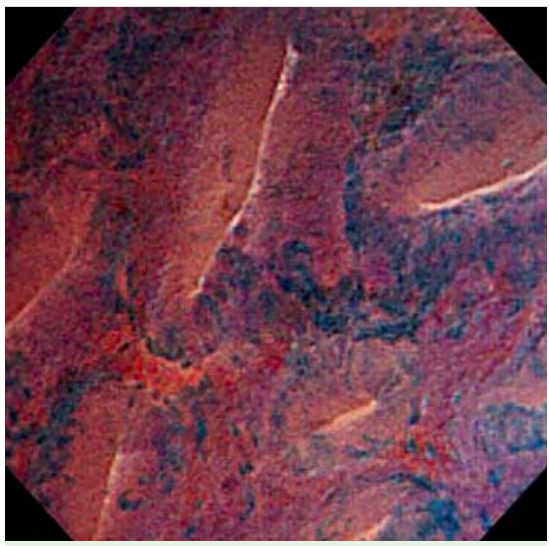

Fig. 4 Endocytoscopy showing fusiform nuclei regularly arranged along the basement membrane, and a smooth and slit-like lumen, remarkably similar to our endocytoscopic images of colonic adenoma and intramucosal neoplasm.

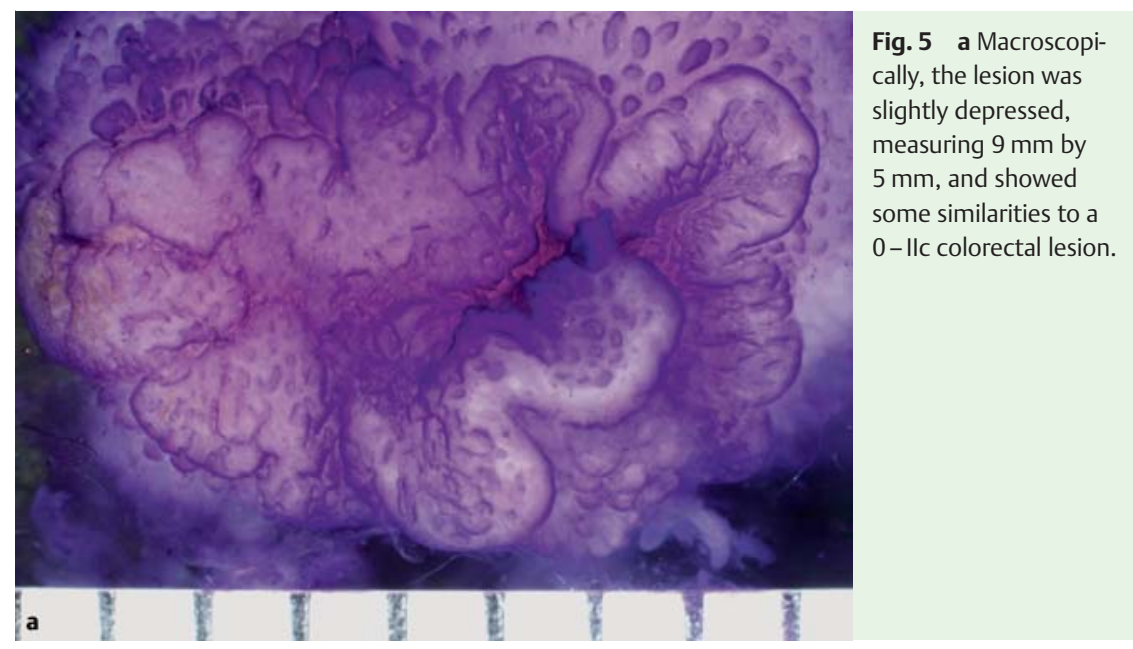




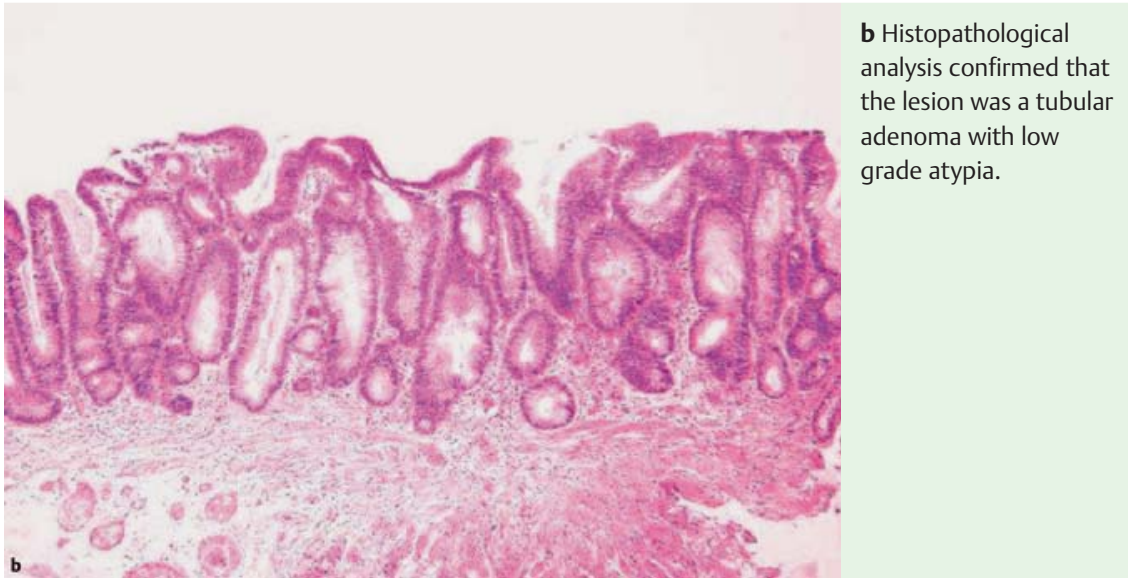

Competing interests: None

Endoscopy_UCTN_Code_CCL_1AC_2AC

H. Shiwaku' ${ }^{1}$ S. Kudo', N. Ikehara', K. Ohtsuka ${ }^{1}$, N. Ogata ${ }^{1}$, K. Wakamura ${ }^{1}$, F. Yamamura ${ }^{1}$, H. Inoue ${ }^{1}$, S. Hamatani ${ }^{2}$

1 Digestive Disease Center, Showa University Northern Yokohama Hospital, Yokohama, Japan

2 Department of Pathology, Showa University Northern Yokohama Hospital, Yokohama, Japan
4 Kudo SE, Takemura O, Ohtsuka K. Flat and depressed types of early colorectal cancers: from East to West. Gastrointest Endosc Clin N Am 2008; 18: 581 - 593

5 Sasajima K, Kudo SE, Inoue H et al. Real-time in vivo virtual histology of colorectal lesions when using the endocytoscopy system. Gastrointest Endosc 2006; 63: 1010-1017

\section{Bibliography}

DOI $10.1055 / \mathrm{s}-0030-1255890$

Endoscopy 2010; 42: E326-E327

(c) Georg Thieme Verlag KG Stuttgart · New York . ISSN 0013-726X

\section{Corresponding author}

\section{S. Kudo}

Digestive Disease Center

Showa University Northern Yokohama Hospital 35-1 Chigasaki-chuo

Tsuzuki-ku

Yokohama 224-8503

Japan

Fax: +45-949-7263

kudos@med.showa-u.ac.jp 